\title{
BUBBLES DURING COVID-19 PERIOD: EVIDENCE FROM THE UNITED STATES USING THE GENERALIZED SUB ADF TEST
}

\author{
Tsangyao CHANG $^{1 *}$ \\ Chen-Min $\mathrm{HSU}^{2}$ \\ Mei-Chih WANG ${ }^{3}$
}

Received: January 2021 | Accepted: February 2021 | Published: April 2021

Please cite this paper as: Chang, T., Hsu, C.M., Wang, M.C. (2021) Bubbles during COVID-19 period: evidence from the United States using the generalized sub ADF test, Holistica Journal of Business and Public Administration, Vol.12, Iss.1, pp.49-56

\begin{abstract}
This study applies the generalized sub ADF (GSADF) tests to investigate whether bubbles exist in the United States markets over the period of 2015-2019, focusing on the COVID-19 period. We use daily Dow-Jones stock price indexes for the first time during the time period of 2015/1/7-2020/3/17. Empirical results demonstrate the existence of bubbles in the US stock market during some subsample time periods. Especially important, we find the third bubble begins from 2020/2/26 and grows gradually, not bursting until recently, a situation that is ongoing in the US market. Our results have important policy implications for investors who attempt to invest in the US stock market

Keywords: Dow-Jones Market Index; SAFDF; GSADF Test; Bubble
\end{abstract}

\section{Introduction}

Since the COVID-19 (coronavirus) outbreak in November 2019, the virus has quickly spread around the world (178 countries affected at the time of this study). As of April, 2020, more than 1.92 million persons have been infected and 119 thousand people have died. Fear of a global economic recession, caused by implications from the virus, triggered a crash in publicly traded securities in many markets. United States markets have witnessed four circuit breakers initiated within 10 days, during March, 2020. This is the largest decline in prices since the 2008 sub-prime crisis. The UN Secretary-General,

\footnotetext{
${ }^{1}$ Department of Finance, Feng Chia University, Taichung, Taiwan, tychang@mail.fcu.edu.tw.

* Corresponding author.

${ }^{2}$ CTBC Business School, Tainan, Taiwan, chenmin@ctbc.edu.tw.

${ }^{3}$ Department of Finance, Providence University, Taichung, Taiwan, wangsona@gmail.com.
} 
Antonio Guterres, stated, on April 2, 2020, that this epidemic is the largest global crisis since World War II.

To stabilize the stock market and avoid further crashes, the FED quickly reduced the prime discount interest rate to near zero-historically unprecedented. No one knows if stock prices have reached a bottom or not. This study represents our attempt to investigate whether bubbles exist in the stock market of the United States using a recently developed GSADF measures. Data comes from the time frames of 2015/1/7-2020/3/17 (covering three-time stock market crisis-August, 2015, October 2018, and March 2020.

Detection of asset bubbles is a topic extensively studied by worldwide scholars. Previous literature shows the test proposed by Phillips et al. (2013) has strong efficacy in testing asset bubbles. In the current study, we utilize the GSADF tests to investigate the existence of bubbles in USA stock markets for the first time using the period of 2015/1/7-2020/3/17. Empirical results demonstrate the existence of bubbles in the USA stock market during some sub-sample time periods.

The remainder of this paper is organized as follows: Section II introduces the methodology that tests for possible bubbles; Section III describes the data and presents our empirical results along with policy implications; Section IV includes our conclusion.

\section{Methodology}

Recently, researchers (Phillips \& Yu, 2011; Phillips et al., 2012) have identified an effective and innovative capacity of dating a wide-range of bubbles in real time. The scope of this approach is that of observing speculative bubbles as they appear, and not just identifying the results after a collapse of the market. These particular aspects can be assessed based on the observation of the behaviour of the phenomenon, especially through the existence of consistent differences between the explosive property of bubbles and the random walk behaviour. In terms of methodology, the new developed interpretation consistent with recursive econometric has the capacity of identifying and analysing the mildly explosive unit roots. The latter functions as a hint for the discovery of bubbles. By taking into consideration typical differences between stationarity and trend stationarity testing procedures that search for the unit root, this particular research directed its scope toward regions of 'no more than' a unit root process or an autoregressive process, where $\delta \leq 1$. In parallel, the model tested by Phillips \& Yu (2011) generates a mildly explosive behaviour through an autoregressive process, that uncovers a root $\delta$ situated in the vicinity of unity. Basically, the idea is of calculating in a recursive manner the unit root tests, with the purpose of assessing the evidence for mildly explosive behaviour in the data. In this particular case, the employed test is a right-sided test, opposite of left-sided tests usually encountered in the calculation of stationarity. As a consequence, the research was constructed under the autoregressive specification by recursive least squares equation: 


$$
x_{t}=\mu+\delta x_{t-1}+\sum_{j=1}^{J} \phi_{j} \Delta x_{t-j}+\varepsilon_{t}
$$

Due to the typicality of the $\mathrm{H}_{0}: \delta=1$, which can be applied to this particular case, but it is less relevant for a stationary alternative then the left-sided tests, researchers (Phillips \& Yu, 2011) decided on the usage of $\mathrm{H}_{\mathrm{a}}: \delta>1$, with $\delta=1+c / k_{n}$, where $c>0, k_{n} \rightarrow \infty$ and $k n / n \rightarrow 0$, having inclinations for mildly explosive cases. Moreover, Phillips \& Yu (2011) tested against the discriminatory power of the equation, since the changes trigger reactions to the process, and determine a shift from the unit root to a mildly explosive root. The same happens when the roles are inversed. The sensitivity of the reaction is significantly higher in the care of right-sided tests, then it is in the left-sided unit-roots that have been tested against stationary alternative. Generally, bubbles have the tendency to collapse periodically; thus, making it difficult for conventional unit root testing to detect the phenomenon. This is the reason why studies (Phillips \& Yu, 2011) presented the usage of supremum of recursive determined Augmented Dickey-Fuller (ADF) t-statistic. Such estimations should allow for the identification of a certain time period where the tendency for explosive property bubble components to dominate in rapport to the asset pricing process. The testing was applied sequentially on particular subsamples, which contained observations from the initial sample to which more observations have been extended until the complete sample was included in the tests. The point in time when the bubble first appeared was estimated as the initial date for the ADF t-statistic. The value of the test was higher in comparison to its corresponding critical value of the right-sided unit root test. The final stage of the speculative bubble was consistent with the moment when the ADF t-statistic value registered below the critical value (Wang et al., 2021).

The sequence of the ADF tests was calculated based on the research proposed by Phillips et al. $(2011,2012)$. This study engaged, though $\hat{\delta}_{\gamma}$, the OLS estimator of $\delta$, and the usual estimator $\hat{\sigma}_{\delta, \gamma}$ for the standard deviation of $\hat{\delta}_{\gamma}$ using the subsample $\left\{x_{1}, x_{2}, \cdots x_{[\gamma T]}\right\}$. The forward recursive ADF test of $H_{0}$ against $H_{a}$ is given by:

$$
\operatorname{SADF}\left(r_{0}\right)=\operatorname{Sup}_{r_{2} \in\left[r_{0}, 1\right]}\left\{A D F_{r_{2}}\right\}
$$

where $A D F=\frac{\hat{\delta}_{t}-1}{\hat{\sigma}_{\delta t}}$. At this point, the testing was computed around the asymmetric interval $\left[r_{0}, 1\right]$. For this particular study, the applications of $r_{0}$ were set around a sample fraction of a reasonable size. Nevertheless, the limitation of the SADF testing was consistent with the fact that the starting point was fixed under the first observation of the sample. The implications determine the presence of two bubbles; the second bubble might be masked by the first bubble. In order to overtake this situation, Phillips et al. (2011) applied a rolling version of the SADF testing, with an interchangeable starting 
HOLISTICA Vol 12, Issue 1, 2021, pp.49-56

window at different points across the sample (Chang et al., 2016). The dimension of the starting window remains fixed, limiting the capacity of the test. As a consequence, Phillips et al. (2012) have suggested to employ the 'Generalized' SupADF (GSADF) testing, under the form of a mechanism to date the interchangeable timeframes. The GSADF diagnostic's formula is based on the sequential right-sided ADF testing, and extends the particular sample sequence to a flexible range. Therefore, contrary to fixing the starting window of the sample, the GSADF test moves the starting and ending points of the sample to a feasible timeframe. The study of Phillips et al. (2012) has demonstrated the performance of the GSADF testing compared to the SADF test. The former has the capacity to expand the sample size in order to detect explosive behaviour of multiple bubble episodes, with very little space for error, even if the sample size is limited. The reason for that resides in the scope of the GSADF testing that covers a wide-range of subsample, and in its potential to detect multiple bubbles in data, outpacing the SADF testing:

$$
\operatorname{GSADF}\left(r_{0}\right)=\sup _{r_{2} \in\left[r_{0}, 1\right], r_{1} \in\left[0, r_{2}-r_{0}\right]}\left\{A D F_{r 1}^{r 2}\right\}
$$

When the regression model includes an intercept and the null hypothesis is a random walk, the limit distribution of the GSADF test statistic is

$$
\sup _{r_{2} \in\left[r_{0}, 1\right], r_{1} \in\left[0, r_{2}-r_{0}\right]}\left\{\frac{(1 / 2) r_{w}\left[w\left(r_{2}\right)^{2}-w\left(r_{1}\right)^{2}-r_{w}\right]-\int_{r_{1}}^{r_{2}} w(r) d r\left[w\left(r_{2}\right)-w\left(r_{1}\right)\right]}{r_{w}^{1 / 2}\left\{r_{w} \int_{r_{1}}^{r_{2}} w(r)^{2} d r-\left[\int_{r_{1}}^{r_{2}} w(r) d r\right]^{2}\right\}^{1 / 2}}\right\}
$$

where $r_{w}=r_{2}-r_{1}$ is a standard Wiener process. Phillips et al. (2013) infer that if the true process is a random walk, the GSADF statistics converge to the standard normal distribution. To obtain the asymptotic critical values by numerical simulations, Phillips et al. (2013) employ a bootstrap methodology to compute the finite sample distributions of the GSADF test. Pavlidis et al. (2012) suggest the method is not affected by a possible explosive root of the determinants of the asset price, while it also provides a datestamping strategy. In the current study, for the first time, we will also use SADF and GSADF tests to investigate bubble occurrence in the USA during the recent time period of 2015/1/7-2020/3/17.

Table 1 Descriptive Statistics of DJ Stock Index Return

\begin{tabular}{cccccccc}
\hline Mean & Median & Maximum & Minimum & Std. Dev. & Skewness & Kurtosis & $\begin{array}{c}\text { Jarque- } \\
\text { Bera }\end{array}$ \\
\hline $4.60 \mathrm{E}-05$ & 0.0002 & 0.03887 & -0.06011 & 0.004743 & -2.44439 & 36.81107 & 63460.43 \\
\hline
\end{tabular}

Source: Authors computation 


\section{Data, Empirical Results, Economic and Policy Implications}

Table 1 reports summary statistics of data sets in terms of returns and results that demonstrate the USA has a monthly mean return of $1.407 \%(0.0000469 * 30)$ with a variance of $0.473 \%$. Based on the Jarque-Bera statistic, from Table 1 , we also observe the USA stock market price returns, which demonstrate a non-normal distribution.

We first apply the right-tailed unit root test of Diba and Grossman (1988). The results have suggested that the stock market in the United States of America showcase explosive behaviour. The application of the GSADF testing was used in this study to identify and observe the occurrence of bubbles in the US stock market, and resulted in obtained critical values by 10000 replications. The research of Phillips et al. (2012) highlighted the capacity of the GSADF test to outperform the SADF test, due to the expansion of the sample size at the occurrence of explosive behaviour in multiple bubble scenarios, without the limitation of error for reduced sample sizes. The GSADF test covers a wide-range of subsamples of the data. This was the argument that determined the application of the GSADF testing in this study, with the scope of identifying the evidence of multiple bubble occurrence in the US stock market. The results are presented in Table 2, where it is strongly visible the evidence for the premise that stock market prices are explosive in some sub-periods. Therefore, we conclude there is significant evidence of exuberance in the stock prices of the USA, allowing us to highlight the possible presence of bubbles ( $\mathrm{Li}$ et al., 2020).

Table 2 Results of the ADF, SADF and GSADF Tests

\begin{tabular}{ccc}
\hline & ADF & GSADF \\
\hline t-Statistic (Prob. $^{*}$ ) & -1.3864 & $2.981130 * * *$ \\
\hline critical values** $^{*}$ level & & \\
99\% level & 0.7257 & 2.715478 \\
95\% level & -0.1029 & 2.354832 \\
90\% level & -0.5512 & 2.127770 \\
\hline
\end{tabular}

Note: ${ }^{* * *}$ denotes significance at $1 \%$ level.

Source: Authors computation

One of the major opportunities offered by the GSADF testing resides in its capacity to underline bubble periods for target assets. In order to locate specific bubble periods, this research compared the SADF backward statistic sequence against the 95\% GSADF critical value sequence, obtained from Monte Carlo simulations with 10000 replications. The results of the date-stamping strategy are observable in Figure 1, over a given period of time for the US stock market. Using the GSADF tests results, the study graphed the estimate of the stock market prices, with $95 \%$ critical value (see Figure 1). The upper curve represents the stock market price index. The middle curve is the $95 \%$ critical value. The bottom curve represents the GSADF statistic. Focusing on the origin and collapse of 
HOLISTICA Vol 12, Issue 1, 2021, pp.49-56

bubbles, the evidence of the bubbles during different sub-sample period in the US stock market is visible

By assessing Figure 1, it can be concluded that the first bubble occurrence happened in the early period of the sample, during 2015/8/21 (a short duration of 1 week). Figure 1 also demonstrates evidence of the second bubble beginning from 2017/10/18 and ending on $2018 / 3 / 22$. The third bubble occurred on $2020 / 22 / 26$, and this bubble grew gradually, however, the third bubble never burst out until a recent period, which is still ongoing in the US stock market. When assessing the bubble periods, it is observable that the bubble occurrences correspond with the recent time periods of stock market crashes: August 2015, October 2018, and March 2020.

The means and methods to fight these ongoing bubbles represent very important aspects for the USA government and investors. As Phillips et al. (2015) argue, this approach is anticipative and can serve as a real-time detector. Policy makers can employ these approaches to estimate risk in the stock market at national macro level. Moreover, investors can avoid investing in the stock market during the time of a bubble and can sell securities before prices drop to avoid losses. Finally, the results of this study can contradict the hypothesis of efficient markets. Nevertheless, testing and predicting the real time occurrence of bubbles is an appealing event for the policy makers, especially due to the opportunity for early detection and correction of possible/probable market failures. At the same time, stakeholders can construct new investment schemes and strategies for portfolio diversification. Conclusively, the present research offers the premises for an instrument of predictability and diagnosis of future stock prices, and for an opportunity of early warning of economic instability, particularly regarding the US stock market. 
Figure 1 GSADF test of the price of DJ Stock Index

GSADF test

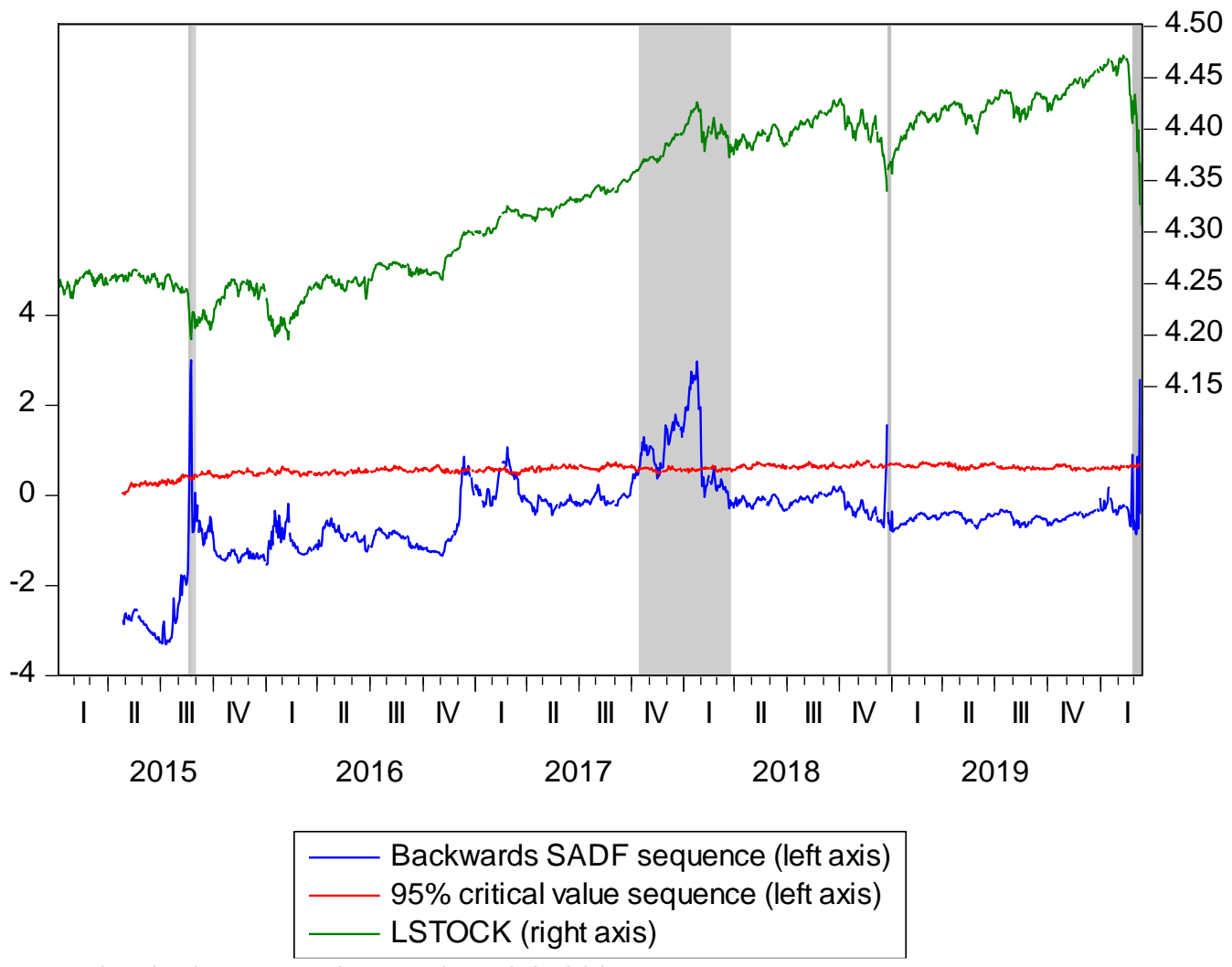

Note: the shadows are sub-periods with bubbles.

Source: Authors computation

Table 3 Bubble Time Periods based on the GSADF Test Results

\section{Bubble Periods}

GSADF 2015/8/21, 2017/10/12 2018/02/02, and 2020/2/26 until right now

Source: Authors computation

\section{Conclusion}

The current research has applied both generalized sub ADF (GSADF) tests to investigate whether bubbles exist in the US stock market, by using data from the recent time periods 


\section{HOLISTICA Vol 12, Issue 1, 2021, pp.49-56}

of 2015/1/7 2020/3/17. The data includes the timeframes of three stock market crashes: August, 2015, October 2018, and March 2020. Empirical results demonstrate that bubbles existed in the USA stock market. The results of this study have important policy implications for policymakers who seek to stabilize the market, and for stakeholders and investors in US securities who want to avoid bubbles.

\section{References}

Chang, T., Gil-Alana, L., Aye, G.C., Gupta, R., Ranjbar, O. (2016) Testing for bubbles in the BRICS stock markets, Journal of Economic Studies, Emerald Group Publishing, vol. 43(4), pages 646-660

Diba, B. T., Grossman, H. I. (1988). 'Explosive rational bubbles in stock prices?' American Economic Review, 78: 520-530.

Funke, M., Hall, S., and Sola, M. (1994). 'Rational bubbles during Poland's hyperinflation: implications and empirical evidence'. European Economic Review, 38: 1257-1276.

Gürkaynak, R. S. (2008). 'Econometric tests of asset price bubbles: taking stock' . Economic Survey, 22: 166-186.

Li, X., Su, C.W., Qin, M., Zhao, F. (2020) Testing for Bubbles in the Chinese Art Market, SAGE Open, doi:10.1177/2158244019901249

Phillips, P. C. B., Shi, S. and Yu, J. (2013). Testing for multiple bubbles: historical episodes of exuberance and collapse in the S\&P 500, Singapore Management University, Working Paper No. 04-2013.

Wang, M.C, Chang, T., Min, J. (2021) Revisit stock price bubbles in the COVID-19 period: Further evidence from Taiwan's and Mainland China's tourism industries, Tourism Economics, https://doi.org/10.1177/1354816620983954

West, K.D. (1987). 'A specification test for speculative bubbles'. Quarterly Journal of Economics, 102: $553-580$. 